\title{
THE SYNTHESIS AND PREDICTION OF BIOLOGICAL ACTIVITY IN SILICO FOR NEW ALKYL DERIVATIVES OF 4-R-3-(MORFOLINOMETYLEN)-4H-1,2,4- TRIAZOLE-5-THIOLES
}

In this work we have conducted the synthesis, the establishment of physical-chemical properties and subsequent Pass-screening of possible biological activity for new alkyl derivatives of 4-R-3-(morfolinometylen)-4H-1,2,4-triazole5-thioles. According to the results of computer prediction it was found that it is advisable to perform in vivo studies for these substances on the ability to use in the treatment of hastrinomy, ulcers, tuberculosis, atherosclerosis and for eradication of Helicobacter Pylori.

Key words: 1,2,4-triazole; synthesis; prediction of biological activity

\section{INTRODUCTION}

Modern scientific research are focused on the implementation of new and safe clinical therapeutic agents in medicine. The nucleus of 1,2,3- and 1,2,4-triazoles is among the most interesting heterocycles which are important fragments of natural products and medicines. Triazoles and their derivatives have a wide range of applications. Yes, they are widely used as antimicrobial, anti-inflammatory, analgesic, antiepileptic, antiviral, antihypertensive, antimalarial, sedatives, antihistamines, anti-TB drugs, etc. $[1,3,9]$. Special attention attracts to the S-derivatives of 1,2,4-triazole. Thus, a detailed biological study allowed to introduce a new domestic veterinary medicine drug "Tryfuzol $1 \%$ solution for injections" (license number: AV-05486-01-14 from 05.08.15) and "Avesstym ${ }^{T M "}$ (license number: AB-05365-01-14 from 07.21.14) which in particular exhibit antiviral, immunomodulating, anti-inflammatory action $[9,10]$. The list of new medicines that contain 1,2,4-triazole core is constantly updating with new promising molecules because the system is low-toxic and pharmacologically active $[3,9]$.

Unfortunately, to investigate experimentally each type of biological effect is almost impossible, since it requires an incredible amount of resources and time [5, 7]. Therefore, to achieve the desired result in the search for new drugs is appropriate and justified the use of computer prediction of biological activity methods.

The aim of research was the synthesis, the establishment of physical-chemical parameters and further computer prediction of the potential biological effects for new alkyl derivatives of 4-R-3-(morfolinometylen)-4H-1,2,4triazole-5-thiole.

(C) Shcherbyna R. O., 2016

\section{MATERIALS AND METHODS}

The research of physical and chemical properties of the obtained compounds is conducted by the methods which are described in the State Pharmacopoeia of Ukraine. The melting point is defined on the automatic device which determines the melting point OptiMelt Stanford Research Systems MPA100. The elemental composition of compounds was found in elemental analyzer Elementar Vario L cube (CHNS) . ${ }^{1} \mathrm{H}$ NMR-spectra of compounds were recorded using a spectrometer Varian Mercury VX-200 $\left({ }^{1} \mathrm{H}, 200 \mathrm{MHz}\right)$, solvent - DMSO- ${ }_{\mathrm{d},}$, internal standard tetrametylsylan (TMS). Chromatography-mass spectral studies were conducted on the gas-liquid chromatograph Agilent 1260 Infinity HPLC equipped with a mass spectrometer Agilent 6120 (ionization in electro-spray (ESI) [2, 4, 6].

As computer program for predicting the biological activity we used a free online system PASS $®$ (Prediction of Activity Spectra for Substances). Chemical structure of compounds introduced by a computer program ACDLABS 12.0 (utility Sketch) as a file with the extension .mol and downloaded to the official website for the range prediction of biological activities located at: http://www.pharmaexpert.ru/passonline/predict.php.

In carrying out the prediction it takes into account the probability of display activity data Pa (Possible activities). These compounds activities which based on the results prediction that had index Pa less than $30 \%$ were excluded from the study results $[5,7]$.

\section{RESULTS AND DISCUSSION}

Experimental Chemical Part

As the starting materials were used 3-(morpholinometylen)-4-ethyl-4H-1,2,4-triazole-5-thiole (1.1) and 3(morfolinometylen)-4-phenyl-4H-1,2,4-triazole-5-thiole (1.2) 
which have been synthesized and described earlier [4]. Further synthesis was carried out by reacting the initial thiols 1.1 and $\mathbf{1 . 2}$ of the relevant halohenalkans (1-brompropan, 1-brombutan, 1-bromheptan, 1-bromhexan, 1-bromoktan, 1-bromnonan, 1-bromdekan) among the i-propanol in the presence of equivalent potassium hydroxide (Fig. 1).

Alkyl derivatives of 4-R-3-(morpholinometylen)4H-1,2,4-triazole-5-thiole (2.1-2.13). To the $0.01 \mathrm{Mol}$ of relevant thiol solution $\mathbf{1 . 1}$ or $\mathbf{1 . 2}$ in $50 \mathrm{ml}$ i-propanol was added $0.01 \mathrm{Mol}$ of potassium hydroxide which was previously dissolved in a minimal amount of distilled water. Heated until thiols dissolve and added $0.01 \mathrm{Mol}$ of appropriate halohenalkan (1-brompropan, 1-brombutan, 1-bromheptan, 1-bromhexan, 1-bromoktan, 1-bromnonan, 1-bromdekan). Heated to boiling on a water heater to $\mathrm{pH}=7$. The resulting solution is filtered, the filtrate evaporated. The synthesized compounds are gray crystalline substances $(2.1,2.4,2.7,2.11,2.13)$, white $(2.2$, $2.3,2.5,2.6,2.8-2.10)$ and light yellow (2.12) color. To analyze the compounds $2.1,2.2,2.4,2.6-2.13$, they were recrystallized from i-propanol, and substances $\mathbf{2 . 3}$ and $\mathbf{2 . 5}$ - from acetone.

Physical parameters of the synthesized compounds are shown in the Tab. 1.<smiles>[R]n1c(S)nnc1CN1CCOCC1</smiles>

1.1, 1.2

The structure of the synthesized compounds in all cases was confirmed by modern instrumental analysis methods $\left({ }^{1} \mathrm{H}\right.$ NMR-spectroscopy, chromatography-mass spectrometry and elemental analysis).

${ }^{1} \mathrm{H}$ NMR-spectra of obtained substances indicate about synthesized compounds conformity to specified formulas (Tab. 2). In carrying out chromatography-mass spectrometric studies there were established individual peaks of synthesized substances, and theoretical calculations of atomic masses correspond to collected data [4].

\section{Experimental Biological Part}

By the result of Pass-screening it was established that the new synthesized o4-R-3-(morfolinometylen)-4H-1,2,4triazole-5-thiole alkyl derivatives may have rather valuable biological effects (Fig. 2). Comparing computer data prediction of biological activity it was found that ethyl radical replacement $(2.1,2.3,2.5,2.8,2.10,2.12)$ to phenyl $(2.2,2.4,2.6,2.7,2.9,2.11,2.13)$ in the $\mathrm{N}_{4}$ atom of 1,2,4-triazole significantly expands the range of biological effects. However, extension of the alkil fragment in hydrocarbon chain virtually doesn't effect on the value of the activity probability (Pa).

Analyzing the results, it was found that all compounds should reduce the secretion of gastrin (value $\mathbf{P a}$ $58,1-47,1 \%$ respectively). This effect can be used in the

$\underset{-\mathrm{KBr}}{\stackrel{\mathrm{KOH}}{\rightleftharpoons}}$

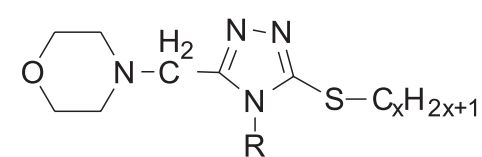

2.1-2.13

Fig. 1. Synthesis of the 4-R-3-(morpholinometylene)-4H-1,2,4-triazole-5-thioles alkyl derivatives. Where: $\mathbf{R}=$ etyl, phenil; $\mathbf{x}=3,4,6,7,8,9,10$

Table 1

PHYSICAL PARAMETERS OF THE 4-R-3-(MORPHOLINOMETYLENE)-4H-1,2,4-TRIAZOLE-5-THIOLES ALKYL DERIVATIVES (2.1-2.13)

$$
\mathrm{O}_{\mathrm{N}-\mathrm{C}^{-}-\mathrm{N}-\mathrm{N}}^{\mathrm{N}} \mathrm{N}_{\mathrm{S}-\mathrm{C}_{\mathrm{x}} \mathrm{H}_{2 \mathrm{x}+1}}
$$

$\mathrm{R}$

\begin{tabular}{|c|c|c|c|c|c|}
\hline Compound & $\mathrm{R}$ & $\mathrm{x}$ & T mel., ${ }^{\circ} \mathrm{C}$ & Gross-formula & Output, \% \\
\hline $\mathbf{2 . 1}$ & etyl & 3 & $105-107$ & $\mathrm{C}_{12} \mathrm{H}_{22} \mathrm{~N}_{4} \mathrm{OS}$ & 90 \\
\hline $\mathbf{2 . 2}$ & phenyl & 3 & $170-172$ & $\mathrm{C}_{16} \mathrm{H}_{23} \mathrm{ClN}_{4} \mathrm{OS}$ & 82 \\
\hline $\mathbf{2 . 3}$ & etyl & 4 & $155-157$ & $\mathrm{C}_{13} \mathrm{H}_{24} \mathrm{~N}_{4} \mathrm{OS}$ & 85 \\
\hline $\mathbf{2 . 4}$ & phenyl & 4 & $156-158$ & $\mathrm{C}_{17} \mathrm{H}_{25} \mathrm{ClN}_{4} \mathrm{OS}^{*}$ & 91 \\
\hline $\mathbf{2 . 5}$ & etyl & 6 & $149-151$ & $\mathrm{C}_{15} \mathrm{H}_{28} \mathrm{~N}_{4} \mathrm{OS}$ & 87 \\
\hline $\mathbf{2 . 6}$ & phenyl & 6 & $150-152$ & $\mathrm{C}_{19} \mathrm{H}_{29} \mathrm{ClN}_{4} \mathrm{OS}^{*}$ & 79 \\
\hline $\mathbf{2 . 7}$ & phenyl & 7 & $151-153$ & $\mathrm{C}_{20} \mathrm{H}_{31} \mathrm{ClN}_{4} \mathrm{OS}^{*}$ & 83 \\
\hline $\mathbf{2 . 8}$ & etyl & 8 & $143-145$ & $\mathrm{C}_{17} \mathrm{H}_{32} \mathrm{~N}_{4} \mathrm{OS}$ & 85 \\
\hline $\mathbf{2 . 9}$ & phenyl & 8 & $148-150$ & $\mathrm{C}_{21} \mathrm{H}_{33} \mathrm{ClN}_{4} \mathrm{OS}$ & 86 \\
\hline $\mathbf{2 . 1 0}$ & etyl & 9 & $144-146$ & $\mathrm{C}_{18} \mathrm{H}_{34} \mathrm{~N}_{4} \mathrm{OS}$ & 84 \\
\hline $\mathbf{2 . 1 1}$ & phenyl & 9 & $147-149$ & $\mathrm{C}_{22} \mathrm{H}_{35} \mathrm{ClN}_{4} \mathrm{OS}$ & 88 \\
\hline $\mathbf{2 . 1 2}$ & etyl & 10 & $115-117$ & $\mathrm{C}_{19} \mathrm{H}_{36} \mathrm{~N}_{4} \mathrm{OS}$ & $\mathrm{C}_{23} \mathrm{H}_{36} \mathrm{~N}_{4} \mathrm{OS}$ \\
\hline $\mathbf{2 . 1 3}$ & phenyl & 10 & $151-153$ & & \\
\hline
\end{tabular}

* - Substances have been obtained as hydrochlorides. 


\section{THE CHEMICAL SHIFTS DATA OF PROTONS IN THE ${ }^{1} \mathrm{H}$ NMR-SPECTRA OF THE 4-R-3- (MORPHOLINOMETYLENE)-4H-1,2,4-TRIAZOLE-5-THIOLE ALKYL DERIVATIVES AND THEIR ELEMENTAL ANALYSIS (2.1-2.13)}

\begin{tabular}{|c|c|c|c|c|c|}
\hline \multirow[t]{2}{*}{ Compound } & \multirow[t]{2}{*}{${ }^{1} \mathrm{H}$ NMR DMSO-d ${ }_{6}, \delta \mathrm{ppm}$} & \multicolumn{4}{|c|}{$\begin{array}{l}\text { Elemental analysis calculated \% } \\
\text { [found \%] }\end{array}$} \\
\hline & & $\mathrm{C}$ & $\mathrm{H}$ & $\mathrm{N}$ & $\mathrm{S}$ \\
\hline 2.1 & $\begin{array}{c}4.49\left(\mathrm{~s}, 2 \mathrm{H},-\mathrm{CH}_{2}-\mathrm{)}, 4.10\left(\mathrm{q}, 2 \mathrm{H},-\mathrm{CH}_{2}-1,2,4 \text {-triazole }\right), 3.44(\mathrm{~m}, 4 \mathrm{H},\right. \\
\text { morpholine), } 3.12\left(\mathrm{t}, 2 \mathrm{H},-\mathrm{CH}_{2} \text { alk), } 2.85\left(\mathrm{~m}, 2 \mathrm{H},-\mathrm{CH}_{2} \text { alk }\right)\right. \\
2.52\left(\mathrm{t}, 3 \mathrm{H},-\mathrm{CH}_{3}-1,2,4 \text {-triazole), } 1.20\left(\mathrm{t}, 3 \mathrm{H},-\mathrm{CH}_{3} \text { alk }\right)\right.\end{array}$ & $\begin{array}{c}53,30 \\
{[53,41]}\end{array}$ & $\begin{array}{c}8,20 \\
{[8,19]}\end{array}$ & $\begin{array}{c}20,72 \\
{[20,75]}\end{array}$ & $\begin{array}{c}11,86 \\
{[11,89]}\end{array}$ \\
\hline 2.2 & $\begin{array}{l}\left.\text { 9.45(s, } 1 \mathrm{H},-\mathrm{NH}^{+}\right), 7.40\left(\mathrm{~m}, 5 \mathrm{H}, \text { phenyl), } 4.54\left(\mathrm{~s}, 2 \mathrm{H},-\mathrm{CH}_{2}-\right), 3.85\left(\mathrm{t}, 2 \mathrm{H},-\mathrm{CH}_{2}\right.\right. \\
\text { alk), } 3.68\left(\mathrm{~m}, 4 \mathrm{H}, \text { morpholine), } 2.64\left(\mathrm{~m}, 2 \mathrm{H},-\mathrm{CH}_{2} \text { alk), } 1.33\left(\mathrm{t}, 3 \mathrm{H},-\mathrm{CH}_{3} \text { alk }\right)\right.\right.\end{array}$ & $\begin{array}{c}54,15 \\
{[54,22]}\end{array}$ & \begin{tabular}{c|c|}
6,53 \\
{$[6,51]$}
\end{tabular} & $\begin{array}{c}15,79 \\
{[15,73]}\end{array}$ & $\begin{array}{c}9,03 \\
{[9,00]}\end{array}$ \\
\hline 2.3 & $\begin{array}{c}4.54\left(\mathrm{~s}, 2 \mathrm{H},-\mathrm{CH}_{2}-\right), 4.15\left(\mathrm{q}, 2 \mathrm{H},-\mathrm{CH}_{2}-1,2,4 \text {-triazole), } 3.50(\mathrm{~m}, 4 \mathrm{H}, \text { morpholine }),\right. \\
3.13\left(\mathrm{t}, 2 \mathrm{H},-\mathrm{CH}_{2} \text {-alk), } 2.68\left(\mathrm{~m}, 4 \mathrm{H},-\mathrm{CH}_{2} \text { alk }\right)\right. \\
2.25\left(\mathrm{t}, 3 \mathrm{H},-\mathrm{CH}_{3}-1,2,4 \text {-triazole), } 1.11\left(\mathrm{t}, 3 \mathrm{H}_{-}-\mathrm{CH}_{3} \text {-alk }\right)\right.\end{array}$ & $\begin{array}{c}54,90 \\
{[54,99]}\end{array}$ & $\begin{array}{c}8,51 \\
{[8,50]}\end{array}$ & $\begin{array}{c}19,70 \\
{[19,67]}\end{array}$ & $\begin{array}{c}11,27 \\
{[11,25]}\end{array}$ \\
\hline 2.4 & $\begin{array}{l}\left.\text { 9.74(s, } 1 \mathrm{H},-\mathrm{NH}^{+}\right), 7.51\left(\mathrm{~m}, 5 \mathrm{H}, \text { phenyl), } 4.66\left(\mathrm{~s}, 2 \mathrm{H},-\mathrm{CH}_{2}-\right), 3.71\left(\mathrm{t}, 2 \mathrm{H},-\mathrm{CH}_{2-}\right.\right. \\
\text { alk), } 3.59(\mathrm{~m}, 4 \mathrm{H}, \text { morpholine }), 2.51\left(\mathrm{~m}, 4 \mathrm{H},-\mathrm{CH}_{2} \text { alk }\right), 1.20\left(\mathrm{t}, 3 \mathrm{H},-\mathrm{CH}_{3} \text { alk }\right)\end{array}$ & $\begin{array}{c}55,50 \\
{[55,74]}\end{array}$ & $\begin{array}{c}6,58 \\
{[6,55]}\end{array}$ & $\begin{array}{c}15,23 \\
{[15,20]}\end{array}$ & $\begin{array}{c}8,72 \\
{[8,70]}\end{array}$ \\
\hline 2.5 & $\begin{array}{c}4.61\left(\mathrm{~s}, 2 \mathrm{H},-\mathrm{CH}_{2}-\right), 4.20\left(\mathrm{q}, 2 \mathrm{H},-\mathrm{CH}_{2}-1,2,4 \text {-triazole }\right), 3.63(\mathrm{~m}, 4 \mathrm{H} \\
\text { morpholine), } 3.07\left(\mathrm{t}, 2 \mathrm{H},-\mathrm{CH}_{2} \text { alk }\right), 1.78\left(\mathrm{~m}, 2 \mathrm{H},-\mathrm{CH}_{2} \text { alk }\right), \\
1.41\left(\mathrm{~m}, 6 \mathrm{H},-\mathrm{CH}_{2-} \text { alk), } 1.07\left(\mathrm{t}, 3 \mathrm{H},-\mathrm{CH}_{3}-1,2,4 \text {-triazole }\right), 0.91\left(\mathrm{t}, 3 \mathrm{H},-\mathrm{CH}_{3} \text { alk }\right)\right.\end{array}$ & $\begin{array}{c}57,66 \\
{[57,81]}\end{array}$ & $\begin{array}{c}9,03 \\
{[9,01]}\end{array}$ & $\begin{array}{c}17,93 \\
{[17,89]}\end{array}$ & $\begin{array}{c}10,26 \\
{[10,27]}\end{array}$ \\
\hline 2.6 & $\begin{array}{c}9.87\left(\mathrm{~s}, 1 \mathrm{H},-\mathrm{NH}^{+}\right), 7.65\left(\mathrm{~m}, 5 \mathrm{H}, \text { phenyl), } 4.59\left(\mathrm{~s}, 2 \mathrm{H},-\mathrm{CH}_{2}-\right), 3.97\left(\mathrm{t}, 2 \mathrm{H},-\mathrm{CH}_{2-}\right.\right. \\
\text { alk), } 3.41(\mathrm{~m}, 4 \mathrm{H}, \mathrm{morpholine}), 1.99\left(\mathrm{~m}, 2 \mathrm{H},-\mathrm{CH}_{2-\mathrm{alk}}\right), 1.35\left(\mathrm{~m}, 6 \mathrm{H},-\mathrm{CH}_{2-\mathrm{alk}} \text {, }\right. \\
0.99\left(\mathrm{t}, 3 \mathrm{H},-\mathrm{CH}_{3} \text { alk }\right)\end{array}$ & $\begin{array}{c}57,48 \\
{[57,31]}\end{array}$ & $\begin{array}{c}7,36 \\
{[7,37]}\end{array}$ & $\begin{array}{c}14,11 \\
{[14,13]}\end{array}$ & $\begin{array}{c}8,08 \\
{[8,10]}\end{array}$ \\
\hline 2.7 & $\begin{array}{c}9.55\left(\mathrm{~s}, 1 \mathrm{H},-\mathrm{NH}^{+}\right), 7.87\left(\mathrm{~m}, 5 \mathrm{H}, \text { phenyl), } 4.63\left(\mathrm{~s}, 2 \mathrm{H},-\mathrm{CH}_{2}-\right), 3.99\left(\mathrm{t}, 2 \mathrm{H},-\mathrm{CH}_{2-}\right.\right. \\
\text { alk), } 3.33\left(\mathrm{~m}, 4 \mathrm{H}, \text { morpholine), } 2.05\left(\mathrm{~m}, 2 \mathrm{H},-\mathrm{CH}_{2} \text {-alk), } 1.47\left(\mathrm{~m}, 8 \mathrm{H},-\mathrm{CH}_{2} \text {-alk }\right)\right.\right. \\
1.05\left(\mathrm{t}, 3 \mathrm{H},-\mathrm{CH}_{3} \text {-alk) }\right.\end{array}$ & $\begin{array}{c}58,45 \\
{[58,64]}\end{array}$ & $\begin{array}{c}7,60 \\
{[7,62]}\end{array}$ & $\begin{array}{c}13,63 \\
{[13,65]}\end{array}$ & $\begin{array}{c}7,80 \\
{[7,78]}\end{array}$ \\
\hline 2.8 & $\begin{array}{c}4.70\left(\mathrm{~s}, 2 \mathrm{H},-\mathrm{CH}_{2}-\right), 4.17\left(\mathrm{q}, 2 \mathrm{H},-\mathrm{CH}_{2}-1,2,4 \text {-triazole }\right), 3.58(\mathrm{~m}, 4 \mathrm{H} \\
\text { morpholine }), 3.10\left(\mathrm{t}, 2 \mathrm{H},-\mathrm{CH}_{2} \text { alk }\right), 1.90\left(\mathrm{~m}, 2 \mathrm{H},-\mathrm{CH}_{2} \text { alk }\right) \\
1.31\left(\mathrm{~m}, 10 \mathrm{H},-\mathrm{CH}_{2} \text { alk }\right), 1.01\left(\mathrm{t}, 3 \mathrm{H},-\mathrm{CH}_{3}-1,2,4 \text {-triazole }\right), 0.87\left(\mathrm{t}, 3 \mathrm{H},-\mathrm{CH}_{3} \text { alk }\right)\end{array}$ & $\begin{array}{c}59,96 \\
{[57,09]}\end{array}$ & $\begin{array}{c}9,47 \\
{[9,49]}\end{array}$ & $\begin{array}{c}16,45 \\
{[16,42]}\end{array}$ & $\begin{array}{c}9,42 \\
{[9,44]}\end{array}$ \\
\hline 2.9 & $\begin{array}{c}9.66\left(\mathrm{~s}, 1 \mathrm{H},-\mathrm{NH}^{+}\right), 7.82\left(\mathrm{~m}, 5 \mathrm{H}, \text { phenyl), } 4.66\left(\mathrm{~s}, 2 \mathrm{H},-\mathrm{CH}_{2}^{-}\right), 3.87\left(\mathrm{t}, 2 \mathrm{H},-\mathrm{CH}_{2-}\right.\right. \\
\text { alk), } 3.38(\mathrm{~m}, 4 \mathrm{H}, \text { morpholine }), 2.00\left(\mathrm{~m}, 2 \mathrm{H},-\mathrm{CH}_{2} \text { alk }\right) \\
1.42\left(\mathrm{~m}, 10 \mathrm{H},-\mathrm{CH}_{2} \text { alk }\right), 1.01\left(\mathrm{t}, 3 \mathrm{H},-\mathrm{CH}_{3-} \text { alk }\right)\end{array}$ & $\begin{array}{c}59,34 \\
{[59,43]}\end{array}$ & $\begin{array}{c}7,83 \\
{[7,85]}\end{array}$ & $\begin{array}{c}13,18 \\
{[13,19]}\end{array}$ & $\begin{array}{c}7,54 \\
{[7,52]}\end{array}$ \\
\hline 2.10 & \begin{tabular}{|c|}
$4.58\left(\mathrm{~s}, 2 \mathrm{H},-\mathrm{CH}_{2}-\right), 4.12\left(\mathrm{q}, 2 \mathrm{H},-\mathrm{CH}_{2}-1,2,4-\right.$ triazole $), 3.49(\mathrm{~m}, 4 \mathrm{H}$ \\
morpholine $), 3.16\left(\mathrm{t}, 2 \mathrm{H},-\mathrm{CH}_{2}\right.$ alk $), 1.86\left(\mathrm{~m}, 2 \mathrm{H},-\mathrm{CH}_{2}\right.$ alk $)$ \\
$1.25\left(\mathrm{~m}, 12 \mathrm{H},-\mathrm{CH}_{2}\right.$ alk $), 1.05\left(\mathrm{t}, 3 \mathrm{H},-\mathrm{CH}_{3}-1,2,4\right.$-triazole $), 0.99\left(\mathrm{t}, 3 \mathrm{H},-\mathrm{CH}_{3-}\right.$ alk $)$ \\
\end{tabular} & $\begin{array}{c}60,98 \\
{[61,15]}\end{array}$ & \begin{tabular}{|c|}
9,67 \\
{$[9,69]$}
\end{tabular} & $\begin{array}{c}15,80 \\
{[15,84]}\end{array}$ & $\begin{array}{c}9,04 \\
{[9,03]}\end{array}$ \\
\hline 2.11 & $\begin{array}{c}9.59\left(\mathrm{~s}, 1 \mathrm{H},-\mathrm{NH}^{+}\right), 7.53\left(\mathrm{~m}, 5 \mathrm{H}, \text { phenyl), } 4.44\left(\mathrm{~s}, 2 \mathrm{H},-\mathrm{CH}_{2}-\mathrm{)}, 3.67\left(\mathrm{t}, 2 \mathrm{H},-\mathrm{CH}_{2-}\right.\right.\right. \\
\text { alk), } 3.29(\mathrm{~m}, 4 \mathrm{H}, \mathrm{morpholine}), 2.05\left(\mathrm{~m}, 2 \mathrm{H},-\mathrm{CH}_{2} \text { alk }\right) \\
1.59\left(\mathrm{~m}, 12 \mathrm{H},-\mathrm{CH}_{2} \text { alk }\right), 0.87\left(\mathrm{t}, 3 \mathrm{H},-\mathrm{CH}_{3} \text { alk }\right)\end{array}$ & $\begin{array}{c}60,18 \\
{[60,29]}\end{array}$ & $\begin{array}{c}8,03 \\
{[8,05]}\end{array}$ & $\begin{array}{c}12,76 \\
{[12,78]}\end{array}$ & $\begin{array}{c}7,30 \\
{[7,32]}\end{array}$ \\
\hline 2.12 & \begin{tabular}{|c|}
$4.62\left(\mathrm{~s}, 2 \mathrm{H},-\mathrm{CH}_{2}-\mathrm{)}, 4.02\left(\mathrm{q}, 2 \mathrm{H},-\mathrm{CH}_{2}-1,2,4\right.\right.$-triazole $), 3.51(\mathrm{~m}, 4 \mathrm{H}$, \\
morpholine), $3.09\left(\mathrm{t}, 2 \mathrm{H},-\mathrm{CH}_{2}\right.$ alk), $1.77\left(\mathrm{~m}, 2 \mathrm{H},-\mathrm{CH}_{2}\right.$-alk), $1.19\left(\mathrm{~m}, 14 \mathrm{H},-\mathrm{CH}_{2-}\right.$ \\
alk), $0.95\left(\mathrm{t}, 3 \mathrm{H},-\mathrm{CH}_{3}-1,2,4\right.$-triazole), $0.81\left(\mathrm{t}, 3 \mathrm{H},-\mathrm{CH}_{3}\right.$-alk $)$
\end{tabular} & $\begin{array}{c}61,91 \\
{[62,05]}\end{array}$ & \begin{tabular}{|c}
9,84 \\
{$[9,86]$}
\end{tabular} & $\begin{array}{c}15,20 \\
{[15,23]}\end{array}$ & $\begin{array}{c}8,70 \\
{[8,72]}\end{array}$ \\
\hline 2.13 & $\begin{array}{c}9.67\left(\mathrm{~s}, 1 \mathrm{H},-\mathrm{NH}^{+}\right), 7.42\left(\mathrm{~m}, 5 \mathrm{H}, \text { phenyl), } 4.51\left(\mathrm{~s}, 2 \mathrm{H},-\mathrm{CH}_{2}-\right), 3.71\left(\mathrm{t}, 2 \mathrm{H},-\mathrm{CH}_{2-}\right.\right. \\
\text { alk), } 3.34(\mathrm{~m}, 4 \mathrm{H} \text {, morpholine }), 2.51\left(\mathrm{~m}, 2 \mathrm{H},-\mathrm{CH}_{2} \mathrm{alk}\right), \\
1.71\left(\mathrm{~m}, 14 \mathrm{H},-\mathrm{CH}_{2} \text { alk }\right), 1.02\left(\mathrm{t}, 3 \mathrm{H},-\mathrm{CH}_{3-} \mathrm{alk}\right)\end{array}$ & $\begin{array}{c}60,97 \\
{[61,15]}\end{array}$ & $\begin{array}{c}8,23 \\
{[8,25]}\end{array}$ & $\begin{array}{c}12,37 \\
{[12,39]}\end{array}$ & $\begin{array}{c}7,08 \\
{[7,10]}\end{array}$ \\
\hline
\end{tabular}

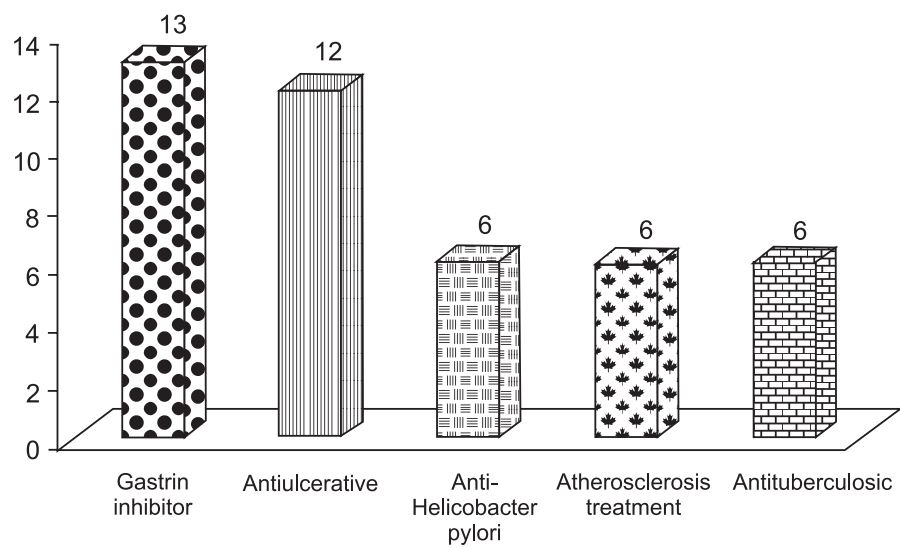

Fig. 2. The results of computer prediction for alkyl derivatives of 4-R-3(morpholinometylene)-4H-1,2,4-triazole-5-thioles 
treatment hastrinomy (Zollinger-Ellison syndrome), which is known to lead to hypertrophy of the gastric mucosa, to enhance its folding, functional stomach glands hyperplasia, main and parietal cells [5]. It is noted that the antiulcer action can show 12 of the 13 synthesized compounds (2.1-2.3, 2.5-2.13, Pa 49,2-35,1\%), and the transition from ethyl substituent $(2.1,2.3,2.5,2.8,2.10,2.12)$ to phenyl $(2.2,2.4,2.6,2.7,2.9,2.11,2.13)$ in the $\mathrm{N}_{4}$ atom of 1,2,4-triazole slightly increases the importance of $\mathbf{P a}$.

In the result of the study it is noted that 6 of the 13 synthesized substances $(2.4,2.6,2.7,2.9,2.11$ and 2.13$)$ can be used in the treatment of tuberculosis (Pa 39,1\%), atherosclerosis (Pa 35,5-35,1 \%) and for the eradication of Helicobacter pylori (Pa 34,2-33,3\%).

\section{CONCLUSIONS}

1. By the study it was received 13 new 4-R-3-(morfolinometylen)-4H-1,2,4-triazole-5-thiole alkyl derivatives structure which in all cases confirmed by modern instrumental analysis methods (1H NMR-spectroscopy, chromatography-mass spectrometry and element analysis).

2. According to the computer prediction results it was found that it is advisable to perform in vivo studies for these substances on the ability to use them in the treatment of hastrinomy, ulcers, tuberculosis, atherosclerosis and eradication of Helicobacter Pylori.

3. The obtained data could help in the future to conduct more focused and purposeful in vivo and in vitro biological action studies of this synthesized compounds class.

\section{REFERENCES}

1. Brenner G. M. Pharmacology / G. M. Brenner, C. W. Stevens. - 4-st ed. - Philadelphia, PA: Elsevier/Saunders, 2012. $-528 \mathrm{p}$.
2. Kazicyna L. A. Application of UV-, YK-, NMR- and MASS-spectroscopy in the Organic Chemistry / L. A. Kazicyna. - 2 ed., revised and expanded. - M.: Publish. Moscow University, 1979. - 236 p.

3. Kumar R. Triazole as pharmaceutical potentials / [R. Kumar, Mohd. Shahar Yar., S. Chaturvedi et al.] // Intern. J. of Pharm. Tech. Res. - 2013. - Vol. 5, № 4. P. 1844-1869.

4. Madden X. T. LC-MS in drug bioanalysis / X. T. Madden.New York: Springer, 2012. - 472 p.

5. Poroikov V. PASS: Prediction of Biological Activity Spectra for Substances / V. Poroikov, D. Filimonov // Predictive Toxicol. Ed. by Christoph Helma. - N. Y.: Marcel Dekker, 2005. - P. 459-478.

6. Saidov T. V. Practical Guide to Molecular Spectroscopy / T. V. Saidov, O. V. Sverdlova. - L.: Publish. SPU, 1995. - 236 p.

7. Shcherbyna R. O. PASS-screening of possible types biological activity 2-(4H-1,2,4-triazole-3-yltio)acetaldehyde derivatives / R. O. Shcherbyna, O. I. Panasenko, Ye. H. Knysh // Med. Chem. - 2011. - № 3 (48). P. 81-84.

8. Shcherbyna R. O. Synthesis and physicochemical properties of 2-((4-R-3-(morfolinometylen)-4H-1,2,4triazole-5-yl)tio) acetic acids / R. O. Shcherbyna, O. I. Panasenko, Ye. H. Knysh, B. O. Varinskiy // Current Issues of Pharmac. and Med. Sci. and Practice. Zaporizhzhya, 2014. - № 3. - P. 18-21.

9. Shcherbyna R. O. Pharmacological activity analysis of the 1,2,4-triazole derivatives / R. 0. Shcherbyna // Pharmac. J. - 2014. - № 4. - P. 145-150.

10. The State Veterinary and Phytosanitary Service of Ukraine. List of registered veterinary products, feed additives, premixes and finished feeds, 2009-2015 // [WWW document]. URL http:// http://www.vet. gov.ua/node/888 (the 11 of January 2016). 


\section{УДК 615.31.014.425:547.792'292-38 \\ Р. о. Щербина \\ СИНТЕЗ ТА ПРОГНОЗУВАННЯ БІОЛОГІЧНОЇ ДІЇ IN SILICO НОВИХ АЛКІЛ-ПОХІДНИХ 4-R-3- (МОРФОЛІНОМЕТИЛЕН)-4Н-1,2,4-ТРИАЗОЛУ-5-ТІОЛІВ}

Проведено синтез, встановлені фізико-хімічні параметри та проведено подальший Рass-скринінг можливих видів біологічної дії нових алкіл-похідних 4-R-3-(морфолінометилен)-4H-1,2,4триазол-5-тіолів. За результатами комп'ютерного прогнозу встановлено, що для даних речовин доцільно проводити in vivo дослідження на здатність до застосовування при лікуванні гастриноми, виразок, туберкульозу, атеросклерозу та ерадикації Helicobacter pylori.

Ключові слова: 1,2,4-триазол; синтез; прогнозування біологічної дії

УДК 615.31.014.425:547.792'292-38

Р. А. Щербина

СИНТЕЗ И ПРОГНОЗИРОВАНИЕ БИОЛОГИЧЕСКОГО ДЕЙСТВИЯ IN SILICO НОВЫХ АЛКИЛ-

ПРОИЗВОДНЫХ 4-R-3 (МОРФОЛИНОМЕТИЛЕН)-4Н-1,2,4-ТРИАЗОЛА-5-ТИОЛОВ

Проведен синтез, установлены физико-химические параметры и проведен дальнейший Passскриннинг возможных видов биологического действия новых алкил-производных 4-R-3 (морфолинометилен)-4H-1,2,4-триазол-5-тиолов. По результатам компьютерного прогноза установлено, что для данных веществ целесообразно проводить in vivo исследования на способность к применению при лечении гастриномы, язв, туберкулеза, атеросклероза и для эрадикации Helicobacter pylori.

Ключевые слова: 1,2,4-триазол; синтез; прогнозирование биологического действия

Адреса для листування:

69035, м. Запоріжжя, пр. Маяковського, 26.

Тел. (061) 233-61-97.

Запорізький державний медичний університет
Надійшла до редакції 25.04.2016 р. 